\title{
Ubicación de riesgos en el trabajo a nanoescala Una visión desde la ética
}

\author{
Carlos Tomás Quirino* Fernando Sancén Contreras**
}

\begin{abstract}
RESUMEN: La investigación y desarrollo (I\&D) en nanociencias y nanotecnología (NyN) entrañan riesgos y beneficios a la sociedad y, desde la ética, deben construirse propuestas que impliquen el control de los riesgos potenciales a la salud y al medio ambiente sin impedir su avance, lo cual conduce a una ética creadora del futuro del hombre, quien actúa sobre sí mismo y sobre su entorno. Este trabajo aborda la nanoética, pretendiendo avanzar en una metodología para ubicar tanto riesgos como beneficios de la I\&D en NyN, específicamente en relación con los nanomateriales en el terreno de la farmacia y la medicina: los nanomedicamentos.

PalABRAS CLAVE: nanotecnología, nanoescala, riesgos, nanoética, ética, nanomedicinas
\end{abstract}

AвsTRAct: Research and development (R\&D) in nanoscience and nanotechnology (NyN) involve risks and benefits to society, and from ethics there should be constructed proposals involving the control of potential risks to health and the environment without hindering their progress, which leads to an ethic that create the future of man, who acts on itself and its environment. This paper addresses nanoethics, trying to advance a methodology to determine both aspects, the risks and benefits of R\&D in N\&N, specifically in relation to nanomaterials in the Pharmacy and Medicine field : nanomedicines.

KEYWORDs: nanotechnology, nanoscale, risks, nanoethics, ethics, nanomedicines

\section{INTRODUCCIÓN}

El surgimiento de nuevas tecnologías como las que se desarrollan actualmente a nanoescala desata con frecuencia un discurso que se autodenomina ético y donde sobresalen los posibles riesgos que implicarían. Tal es el caso de la nanotecnología: con frecuencia se la ve solamente bajo la óptica del riesgo, sin considerar en toda su dimensión los beneficios que aporta y podría aportarle en el futuro a la sociedad. Lo anterior exige realizar un esfuerzo para mostrar que las tecnologías emergentes en general y la nanotecnología en particular entrañan tanto riesgos como beneficios. Conviene centrar el debate sobre estas cuestiones hacia la construcción de propuestas para impulsar la investigación y el desarrollo (I\&D) de las nanotecnologías de tal manera que los riesgos que esto implica potencialmente para la salud y el medio ambiente puedan controlarse. Esto, desde luego, tiene rasgos propios de una empresa imposible, debido a que se trata de regular algo que apenas se está haciendo lo cual tiene un amplio margen desconocido, especialmente en lo que se refiere a los efectos que producirá una vez que exista. No obstante lo anterior, es necesario seguir de cerca el desarrollo de las nanotecnologías para ir conociendo, justamente, sus potencia-

\footnotetext{
* Coordinador de la maestría en ciencias farmacéuticas, División de Ciencias Biológicas y de la Salud, UAM Xochimilco. Miembro del grupo coordinador de la Red Nanociencias UAM. UAM Xochimilco.

** Coordinador del Área de Investigación Polemología y Hermenéutica. UAM Xochimilco. Miembro del grupo coordinador Red Nanociencias UAM.
} 
lidades y así poder impulsarlas así como sus riesgos para conseguir controlarlos, por tanto, se hace necesario ubicar y dimensionarlos con el objetivo de ampliar el beneficio que traerán para el hombre actual. A las nanociencias y nanotecnologías (NyN) se les reconoce una fuerza expansiva en el desarrollo científico y tecnológico mundial de tal magnitud que se las ve como el origen de una revolución completa en nuestra vida actual y en la forma como concebimos al mundo.

El concepto de riesgo tiene una proyección necesariamente hacia la ética en la medida en que debe ser evitado; pero, de la misma forma, el concepto de beneficio proyecta hacia ella porque es un bien a conseguir; esto último, sin embargo, en el discurso común, generalmente no se vincula con la ética.

Nuestro trabajo se ubica en el terreno de la ética y desde ella aborda la nanoética con la pretensión de avanzar hacia una metodología que permita situar tanto los riesgos como los beneficios de la I\&D en NyN, específicamente en lo que se refiere al manejo de nanomateriales en el terreno de la farmacéutica y la medicina.

\section{LA NANOÉTICA}

La ética es una disciplina filosófica tan compleja y abarcante como lo es el análisis integral de la acción humana. Dicha complejidad no sólo se basa en las características propias del ser humano y de su actuar, en los antecedentes, sus motivaciones, sus aspiraciones. La complejidad del análisis ético alcanza también al lugar y al medio en que se realiza. Dada esta complejidad de la ética, al considerar su dimensión aplicativa, se hace necesario precisar los elementos que intervienen; conviene elaborar un mapa inicial donde sea posible ubicar fácilmente un objeto concreto de estudio. Tal es el caso de la nanoética, donde vemos necesario establecer una ruta conceptual que sea útil para ubicar en el amplio espectro de las NyN el posible riesgo que los materiales ahí obtenidos generan para la integridad del ser humano y de su entorno.

La ciencia y la tecnología (CyT) que se desarrollan actualmente a nanoescala, constituyen un contenido determinante de la actividad científica actual no sólo por los avances que aportan en lo que se refiere a la explicación racional del mundo, sino por el impacto que tienen hacia la sociedad en su conjunto. La CyT, por tanto, no pueden ser ajenas a la misma sociedad; estamos ya lejos de la visión de la ciencia como una "búsqueda desinteresada de la verdad" que justificaba el aislamiento al interior de la comunidad científica y respecto de la sociedad. La ciencia está dejando su "torre de marfil" en la medida en que se va haciendo consciente de las aplicaciones tecnológicas que genera o que podría generar, aun sin la participación del científico que en la investigación básica haya contribuido a enriquecer la explicación del mundo material en el que se desenvuelve nuestra vida. Las NyN son un caso en el que se puede apreciar claramente cómo la explicación y la manipulación de la materia a nanoescala está modificando los procesos industriales, los bienes terminados que están al alcance de los consumidores, y, desde luego, a la sociedad misma. Esto hace ver que la nanoética no puede limitarse a los riesgos que posiblemente generaría. La razón puede verse, primero, porque no son reales dichos riesgos, y, luego, porque llevarían a la negación misma de la I\&D bloqueando los beneficios que pueden aportar. Sin negar los riesgos, es necesario dimensionarlos para controlarlos. Pero también es indispensable tomar en cuenta el poder transformador de la CyT. En todo esto la ética tendrá el papel de orientarlas para conformar el bien colectivo de la sociedad. Esto nos aleja de una ética estrictamente basada en la negación o limitación de la acción humana 
y nos acerca a una ética creadora del futuro del hombre, quien actúa sobre sí mismo y sobre su entorno.

En general, podemos decir que las NyN, más allá de su origen y naturaleza científica, constituyen una cuestión ética que atañe a todos los miembros de nuestra sociedad, porque el papel que cada uno de ellos desempeña está determinado por los resultados de la CyT, específicamente de las NyN.

\section{Una ética que se proyecta hacia el futuro}

Pensar en una ética vuelta hacia la conformación del futuro no es novedad. Siempre se ha reconocido que la acción humana tiende a la obtención de un bien generalmente no presente, sino futuro, y que el resultado de dicha acción forma o constituye al sujeto que actúa. Sin embargo, se ha insistido también en que la acción humana debe ajustarse a normas ya establecidas a las que se les otorgan rasgos absolutos e incuestionables. La CyT han hecho ver en las últimas décadas que ciertas normas consideradas absolutas han sido modificadas gracias al avance de la ciencia, tanto porque se demuestra que algo previamente considerado verdadero en realidad es falso(v.g., la pérdida del centro con la teoría heliocéntrica), como porque se incorporan en la sociedad nuevos instrumentos, equipos, bienes, derivados de la CyT, que posteriormente generan cambios en las costumbres y valores sociales (v.g., la familia, la propiedad, el papel de la mujer en la sociedad, etc.). Gracias a esta modificación de normas antes consideradas como absolutas, surge la necesidad de pensar una ética del devenir en la que más que ajustar la acción del hombre a normas consideradas como absolutas se asuma desde dicha acción la responsabilidad de la futura realidad que se construye a partir del actuar humano. Es desde esta perspectiva que consideramos a las NyN y los riesgos posibles que entraña su desarrollo y aplicación en bienes útiles para la sociedad.

Así, dado el amplio espectro que cubren las NyN, la nanoética no consiste en denunciar el riesgo, sino que tiende a precisar su ubicación y dimensión para su posible manejo. Es así como la ética puede legitimar y sustentar el predominio de las NyN como un nuevo campo científico y tecnológico que traerá grandes beneficios a la sociedad, pero también como un medio de contención o conducción razonada que sea capaz de medir el impacto social de la ciencia y tecnología en este campo, para realizar los cambios de dirección que sean considerados como necesarios.

En este contexto, se trata de favorecer y no de inhibir a la I\&D en el campo de las $\mathrm{NyN}$, de modo que, como se reconoce en algunos círculos, se busca orientar los estudios éticos en un sentido positivo, es decir, darle una dirección a la I\&D en el campo de las NyN, hacia la búsqueda de competencias éticas que contribuyan al desarrollo científico y tecnológico crítico, responsable y "bueno" de la nanotecnología, así como de sensibilizar al grueso de la sociedad sobre la necesidad de que esta actividad, junto con sus productos, debe ser vista siempre sobre la base de un análisis crítico de la relación riesgo-beneficio que tienen o tendrán en la sociedad.

Para algunos se trata de que en la nanoética prevalezca el sentido positivo y no el negativo de la ética, reconocido este último como una visión limitada al desarrollo de competencias o estudios orientados a identificar y eludir los efectos dañinos que pueden traer como secuela las NyN. Nuestra propuesta es no repetir la mala experiencia de la biotecnología y los productos genómicos (transgénicos), donde sólo a posteriori se reflexionó sobre sus efectos sobre el ser humano y el medio ambiente. Se pretende que la nanoética permita desarrollar una metodología mediante la cual se pueda 
realizar una evaluación de los riesgos implicados en la propia actividad y por la exposición del hombre y el medio ambiente a las nanopartículas y los productos que las contengan.

\section{El riesgo de los nanomateriales}

Se define un riesgo como una consecuencia incierta de un acontecimiento o actividades respecto a algo que la sociedad considera como valioso. El riesgo tiene dos componentes: la posibilidad de consecuencias potenciales, y el rigor o fuerza de dichas consecuencias. Además, el riesgo no se refiere solamente a las consecuencias físicas, sino también al impacto financiero, sobre las inversiones, las instituciones, la cultura y el impacto psicológico. (Kshitij Aditeya Sing, Institute of Nanotechnology)

Aunque se habla de riesgo y de azar (accidente) como si fueran sinónimos, no lo son: el azar [fuente de peligro] describe la posibilidad de daño; denota la propiedad inherente de un factor de riesgo o procesos relacionados, donde un riesgo expresa el efecto posible. El riesgo se refiere a las consecuencias de algo respecto a lo considerado valioso. De acuerdo con su origen, se le clasifica como agentes físicos, agentes químicos, agentes biológicos, fuerzas naturales, fuerzas sociales, y accidentes complejos.

En general, se dice que existe un riesgo cuando se percibe la posibilidad de que ocurra un daño. En las finanzas se mide dicho riesgo, y ante un préstamo o una inversión se paga una cantidad extra por la contingencia de que no se pague la cantidad prestada. Algo semejante ocurre en las tecnologías emergentes, donde el riesgo se ubica especialmente en el daño que puedan provocar en el ser humano o en el medio ambiente. A este respecto se habla de "manejo del riesgo" para expresar la necesidad de conducir la investigación científica y tecnológica frente a la certeza de un daño. Esto tiene graves problemas en su aplicación porque la valoración científica de la presencia del riesgo no se conoce cuando se desarrolla la investigación.

No obstante esta grave dificultad, su novedad y el consecuente desconocimiento de los nanomateriales en el universo de la CyT, se pueden ubicar posibles riesgos en la exposición del medio ambiente y del ser humano a los nanomateriales y nanotubos. Como afirma Roland Clift (2006: 145) el posible riesgo de una sustancia depende de sus propiedades. Hay que señalar que ni el riesgo de los nanomateriales mismos, ni de sus propiedades son algo nuevo, porque se presentan ya comúnmente en acontecimientos naturales como erupciones volcánicas, en la combustión, e incluso en forma de virus. Sin embargo, en lo que se refiere a la obtención, manejo y utilización de nanopartículas, el ser humano está expuesto a ser afectado por las ellas de tres formas: por la respiración, por la exposición de la piel, y por la ingestión. Es posible avanzar en esta difícil tarea: la de valorar los riesgos posibles, precisando los elementos que por su naturaleza están presentes en todo riesgo. Posteriormente será necesario ubicar el riesgo en toda la ruta por la que se transita, desde la obtención, hasta la deposición de las nanopartículas, tal como se puede observar en la tabla 1.

Además de lo anterior, y dado que el manejo de la materia a nanoescala genera un comportamiento diferente por las nuevas propiedades que obtiene, es necesario ubicar el riesgo no solamente en los nanomateriales mismos, sino también en sus propiedades, tales como su conductividad, volatilidad, corrosividad, entre otras. De esta manera, será posible aplicar los criterios de la ética a condiciones precisas. 
TABLA 1. Elementos para valorar el riesgo de los nanomateriales

\begin{tabular}{|c|c|}
\hline Valoración del riesgo & $\begin{array}{l}\text { Identificación del riesgo: identificación de la ca- } \\
\text { pacidad propia de una sustancia para cau- } \\
\text { sar efectos adversos sin tomar en cuenta la } \\
\text { probabilidad o severidad de dichos efectos. } \\
\text { Caracterización del riesgo: evaluación cuantitativa } \\
\text { de los efectos adversos generados por la exposición } \\
\text { a una sustancia química. }\end{array}$ \\
\hline Valoración de la exposición al riesgo & $\begin{array}{l}\text { Evaluación cualitativa de la posible exposición de } \\
\text { los humanos y del medio ambiente a la sustancia } \\
\text { (origen del riesgo). }\end{array}$ \\
\hline Caracterización del riesgo & $\begin{array}{l}\text { Estimación cualitativa de la probabilidad de que } \\
\text { ocurra un efecto adverso, y de su fuerza y duración } \\
\text { bajo condiciones definidas de la exposición. }\end{array}$ \\
\hline
\end{tabular}

Fuente: Roland Clift, "Risk management and regulation in an emerging technology", en Hunt G. y Mehta M. (eds.), Nanotechnology. Risk, ethics and law, Earthscan, 2006: 144.

De manera general, asumimos que el riesgo potencial de las NyN se encuentra en la I\&D por la que se obtienen, manejan, utilizan y deponen los nanomateriales. Nos referimos a la construcción, a la aplicación, al uso, y a la dispersión de las nanopartículas en general tanto en substancias o aparatos específicos, como en el medio ambiente.

Desde esta perspectiva, distinguimos dos grandes horizontes donde se hace presente el riesgo derivado de los nanomateriales: el mismo proceso de su manufactura, y su presencia física, nunca perceptible sensiblemente, pero incorporada a la vida del ser humano. Conviene destacar que el riesgo se refiere siempre al bienestar humano o al de su entorno.

\section{MANUFACTURA DE LOS NANOMATERIALES}

En el ámbito de su manufactura, los nanomateriales, al igual que los insumos precursores y los mismos procedimientos o procesos, implican un riesgo potencial en el momento de su elaboración, lo mismo aplica para su utilización cuando se generan productos más complejos de mayor valor agregado orientados al consumo, así como al uso de dichos productos, y en su presencia como principios activos en el medio ambiente. Esta dimensión del riesgo se refiere específicamente a los nanomateriales desde su obtención hasta su aprovechamiento y luego en su manejo, una vez que termina su vida útil. En este proceso, el individuo está expuesto de manera diversa. En efecto, el individuo humano está expuesto a los nanomateriales, primero por razones de su ocupación, generalmente en el laboratorio o en el medio industrial donde se obtienen y manipulan las nanopartículas.

Pero también la presencia de los nanomateriales puede representar un riesgo para la población en su vida diaria cuando los primeros se incorporan en la elaboración de productos pudiendo llegar al organismo por su presencia en el aire (inhalación), por su contacto físico (vía dérmica), o por su ingestión en alimentos sólidos o 
líquidos, incluida el agua. Esto aplica también, y de modo muy especial, a los medicamentos administrados directamente al organismo humano.

Riesgo en el medio ambiente

Los nanomateriales se hacen presentes en el medio ambiente. Es probable que ahí se encuentren como riesgo potencial en el agua, en el aire, en los sedimentos y en el suelo, pudiendo afectar al hombre directa o indirectamente a través del impacto nocivo en el medio físico, la fauna, o la flora.

En general, el riesgo eventual de la presencia de los nanomateriales se da tanto en el proceso de su obtención en el que se manipula la materia, como en el empleo de dichos nanomateriales en cosméticos, alimentos, medicamentos, textiles, aparatos diversos de telecomunicación o de diagnóstico, como insumos para la industria de materiales, la farmacéutica, la química, entre otros muchos. (véase la figura 1).

Otra forma de ubicar el posible riesgo tanto en la I\&D de las NyN como en la industria, proviene del tipo de nanomateriales que actualmente existen, y de otros que seguirán siendo desarrollados, pues, como se sabe, los nanomateriales manifiestan nuevas propiedades, mismas que les otorgan características que hasta entonces no poseían. Hoy se puede precisar el riesgo a partir de las características propias que tienen los nanomateriales manejados como nanotubos, o como 'nanofulerenes', o 'nanobuckyballs', por mencionar algunos.

Para completar esta visión de la nanoética, es necesario mencionar también otra forma de abordar el tema del posible riesgo derivado de los nanomateriales. Nos referimos al criterio del ámbito donde se desarrolla actualmente la investigación de las

FIGURA 1. Ámbito de posible riesgo a partir de nanomateriales

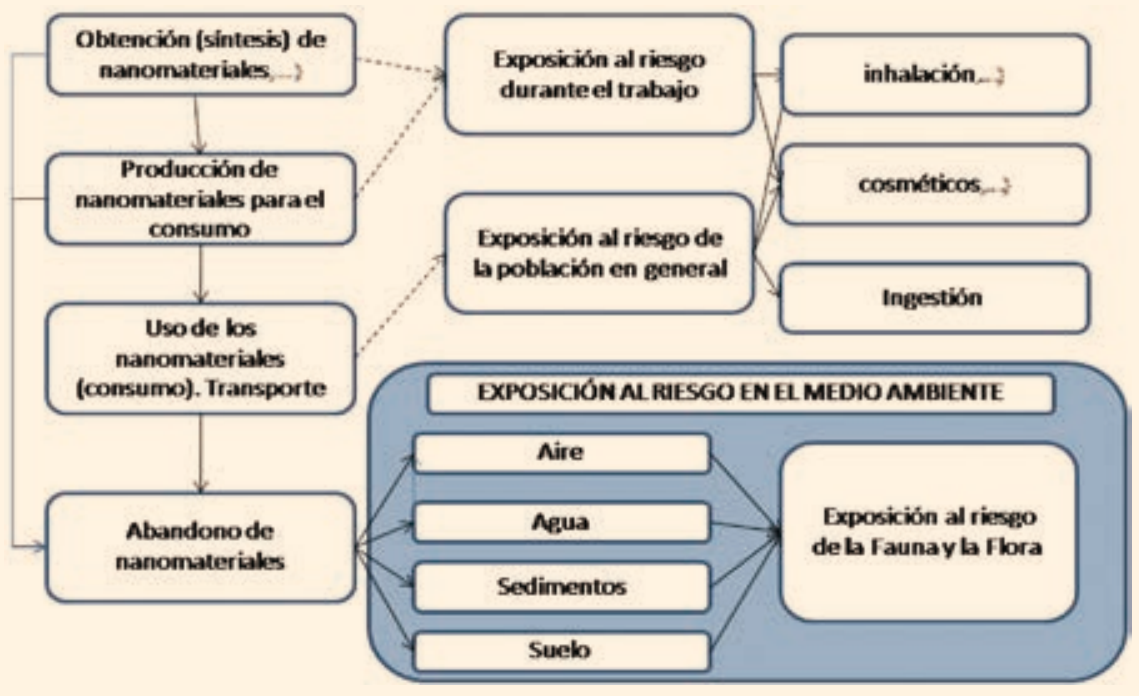

Fuente: Traducida y adaptada de FramingNANO, Mapping Study on Regulation and Governance of Nanotechologies, enero 2009: 51. 
NyN para su aplicación inmediata: la nanobiotecnología, la nanomedicina, la nanocosmetología, así como las técnicas de comunicación.

\section{Desarrollo y liberación de fármacos}

Nos referiremos ahora a la dimensión del riesgo de la exposición a los nanomateriales en la nanobiotecnología, específicamente en la innovación para la administración y liberación de medicamentos utilizando los nanomateriales.

La liberación de fármacos es una de las principales aplicaciones de la nanotecnología en medicina. Los fármacos incorporados o contenidos en nanotransportadores dirigidos permiten un nuevo grado de especificidad y eficacia en la administración y liberación de medicamentos.

Una característica de los nanomedicamentos es su complejidad, porque en ellos se pretende combinar diversas características. En efecto, los nanomedicamentos podrían liberar moléculas terapéuticas directamente traspasando las barreras biológicas llevando consigo moléculas específicas o cargas en su caparazón o coraza exterior. Al mismo tiempo, esta coraza podría incluir reactivos como anticuerpos que alcanzaran objetivos específicos ( $v . g$. ., moléculas típicas del cáncer o en el caso de la inflamación de tejidos). El portador podría ser elaborado de material fisiológicamente estable que solamente desintegrara o disolviera su objetivo (o bien al recibir una señal externa, enviada por el personal médico). Esto permitiría la liberación de fármacos altamente potentes que de otra manera tendrían efectos adversos cuando fueran administrados sistémicamente o de aquellos que no pueden ser administrados éxitosamente por los métodos convencionales. Las pruebas clínicas ya comenzaron para muchos nanomedicamentos, y aunque numerosos fármacos actualmente disponibles para el público no son distribuidos explícitamente en el mercado como nanomedicinas, podrían ser considerados como nanofármacos.

El beneficio de los nuevos sistemas de liberación de medicamentos para los pacientes consiste en menores efectos colaterales, una mayor eficacia y la posibilidad de tratar enfermedades que actualmente no lo pueden ser de manera efectiva (como es el caso del cáncer). Algunos ejemplos de los sistemas de liberación de fármacos son las nanoemulsiones y las nanosuspensiones, anticuerpos conjugados, sistemas multicompuestos, agentes liposomales anticáncer, y nanoprecipitados. Los métodos pueden ser combinados (v.g., aislando tejidos dañados del sistema vascular y depositando localmente una sustancia que induce la muerte de la célula). También harían posibles nuevas formas de terapia (v.g., una nueva forma de termoterapia: partículas de hierro u otros elementos que pueden llegar a células malignas en el cáncer de cerebro y crear calor local cuando el médico pone al paciente en un campo magnético externo, mejorando con ello los efectos de la quimio $\mathrm{y}$ radio terapia).

Junto con los materiales farmacogenéticos y farmacogenómicos, la liberación de fármacos a través de la nanotecnología puede ayudar a obtener una terapia personalizada a base de medicamentos, una vez que los mecanismos de acción del medicamento y la respuesta del organismo se entiendan mejor.

Otra posibilidad será la combinación de biosensores miniaturas que almacenen unidades de fármacos para la implantación en donde se requieran; un sistema integrado a control remoto que regule la liberación de fármacos, que reciba retroalimentación del sensor, y transmita 
actualizaciones para su operación. (Traducción de la Opinión 21 del Grupo europeo de ética en la ciencia y en las nuevas tecnologías para la Comisión Europea, enero de 2007).

Este breve panorama de la naturaleza y propiedades de los nanomedicamentos remite primero a la ubicación del riesgo conforme al mapa ya trazado para las nanopartículas. Hay que añadir, sin embargo, la complejidad que le añade el hecho de que dichos nanomateriales están orientados a modificar el funcionamiento de células que forman parte de órganos complejos, los cuales, a su vez, componen al organismo de animales superiores y, finalmente, del hombre. Cobra aquí relevancia la nanomedicina que incluye la liberación y administración de fármacos, y también la construcción del código de ética para la nanomedicina.

En este contexto, conviene mencionar el Código de Conducta adoptado por la Unión Europea para los responsables de la investigación en NyN que consta de siete principios: ${ }^{1}$

1. Comprensibilidad: la investigación en NyN debe ser comprensible para el público. Deben respetarse los derechos fundamentales y orientarse hacia el bienestar de individuos y sociedad en su diseño, implementación, distribución y uso.

2. Sustentabilidad: la investigación en $\mathrm{NyN}$ debe ser saludable (safe), ética, y contribuir al desarrollo sustentable. No debe causar daños o afectar a la población, ni a animales, plantas, o el entorno, en el presente y en el futuro.

3. Precaución: la investigación en NyN debe ser realizada conforme al principio de precaución, anticipándose a posibles impactos sobre el medio ambiente y la salud, tomando las debidas precauciones, proporcionalmente al nivel de protección, pero promoviendo el progreso para beneficio de la sociedad y del medio ambiente.

4. 'Inclusividad': las investigaciones en $\mathrm{NyN}$ deben ser guiadas por los principios de apertura a los interesados, transparencia y respeto conforme al legítimo derecho de acceso a la información. Deben, asimismo, permitir la participación de todos los interesados directa o indirectamente.

5. Excelencia: la investigación en $\mathrm{NyN}$ debe alcanzar los mayores estándares científicos incluyendo la integridad y honestidad en la investigación y buenas prácticas de laboratorio.

6. Innovación: la dirección de las actividades de investigación en NyN deben estimular un alto grado de creatividad, flexibilidad y habilidad para planificar la innovación y el crecimiento.

7. Rendición de cuentas: los investigadores y las organizaciones de investigación en NyN deben permanecer abiertas para la sociedad respecto a los impactos de su trabajo sobre la salud humana y el medio ambiente.

UBICACIÓN DE RESPONSABLES

Además de la ubicación física del riesgo derivado de la obtención y manejo de nanomateriales, conviene ir a la dimensión de la acción y considerar necesariamente a quienes

\footnotetext{
1 European Comission, Code of conduct for responsible nanosciences and nanotechnologies research, <http://europa.eu/rapid/pressReleaseAction.do?reference=IP/08>, 8 de febrero de 2008. Traducción de los autores.
} 
son responsables de sintetizar, construir, distribuir y comercializar los nanomateriales. Nos referimos a la responsabilidad moral de quienes están manipulando la materia a nanoescala, primero hacia sí mismos, y luego hacia su propio entorno. Esta responsabilidad tiene dos vertientes: 1) hacia el individuo, en la medida en que tenga conciencia de que su propia acción ha de estar basada en sus propios principios sin coacción alguna; 2) hacia "lo otro", es decir, la conciencia de que la acción que libremente decida utilizar tiene un impacto sobre los entes con los que comparte su entorno. Existe, por tanto, una responsabilidad hacia sí mismo respecto de la naturaleza de su acción y respecto de la forma como persigue el objetivo que se ha fijado. Otra dimensión de esta responsabilidad se refiere a su entorno bajo la certeza de que su propio bien, su existencia misma, depende de los entes que conforman su entorno natural y social. ${ }^{2}$

La dimensión moral, por otra parte, se refiere no sólo a los nanomateriales vistos como elementos, sino que abarca también a los compuestos físicos o químicos y a los aparatos o bienes que los contienen y que son utilizados por el hombre en su existencia cotidiana.

De esta forma podemos ubicar la responsabilidad en las personas que inciden en la generación de un posible riesgo a la salud humana y al medio ambiente, riesgo proveniente de los nanomateriales: industriales, administradores de empresas industriales y comerciales, agentes financieros, gobernantes, personal de las universidades, profesores y estudiantes en laboratorio, investigadores (titulares, asociados, técnicos, etc.), agentes de las oficinas de patentes, entre otros muchos.

FIGURA 2. IRGC Cuadro de manejo de riesgos.

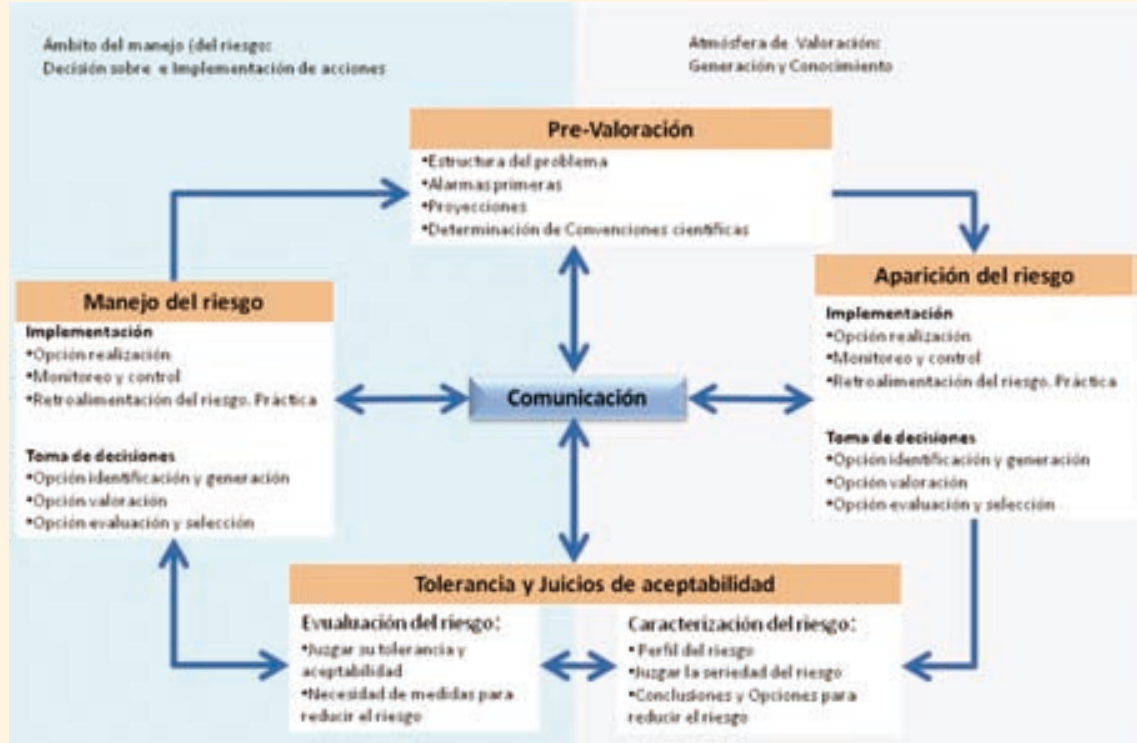

Fuente: White paper on Risk Governance - Towards an Integrative Approach: p. 13. Traducción propia

\footnotetext{
2 Véase a este respecto, Ernst Tugendhat, Egocentricidad y mística. Un estudio antropológico. Traducción del orignal alemán, Egozentrizität und Mystik, por Mauricio Suárez Crothers, Barcelona: Gedisa, 2004.
} 
Estas formas diversas de ubicar el análisis y la responsabilidad del posible riesgo derivado de los nanomateriales cuentan con una orientación que desde la visión de nuestra sociedad cobran relevancia y generan, aunque inicialmente, una norma o costumbre que podría verse como ética. Nos referimos a la manera en que se conciben actualmente las NyN. En efecto, éstas son consideradas como un factor indiscutible de cambio no sólo en los aparatos que utilizamos para nuestra vida diaria, sino también porque afectan necesariamente a la sociedad. Se las reviste de un "halo" transformador de la sociedad. Desde esta perspectiva, podemos visualizar una primera exigencia originalmente de los científicos y tecnólogos que conocen de manera más cercana los fenómenos que sirven de base para generar los productos derivados de las NyN, quienes están obligados a informar de los efectos que tendrán los resultados de los trabajos que realizan y también de los que piensan realizar. De esta información se sigue, necesariamente, el ejercicio de la responsabilidad moral de inversionistas, políticos, asesores, industriales, etc. respecto de los resultados que obtendrán del trabajo de I\&D a nivel de nanoescala.

Hay que reconocer, sin embargo, que en el terreno de la toxicidad, riesgo concreto de los nanomateriales, existe aún un desconocimiento que impide ahondar y precisar el trabajo de la nanoética.

Ante el panorama paradójicamente incierto y a la vez transformante de las $\mathrm{NyN}$, su I\&D deben moverse entre la creatividad y la precaución. Sin embargo, el estudio a profundidad de los riesgos a evitar no puede ser práctica cotidiana para los investigadores.

En nuestro medio, la principal obligación ética consiste en el desarrollo de las NyN orientado a la solución de nuestros problemas más acuciantes (hambre, pobreza, desempleo). Moralmente estamos obligados a obtener nuevas formas de organización, nuevas herramientas, nuevos equipos que sabemos podemos lograr con las NyN. Respecto al riesgo, tenemos también la obligación de proteger la salud y el medio ambiente de nuestro país de posibles daños causados por la I\&D en NyN. Ante esto, proponemos adoptar en principio las medidas que sobre riesgos en NyN adopten en la práctica los países de la UE o nuestros socios del TLCAN. Esto podría ser difundido previo análisis responsable a realizar por el Centro Nacional de Metrología (Cenam), el que a su vez solicitaría colaboración colateral de los responsables de los proyectos de I\&D en NyN; quizás monitoreando en sus laboratorios los aspectos que el Cenam considerara pertinentes.

El grueso de los recursos nacionales, en consecuencia, deberán estar orientados a las áreas de I\&D en N\&N que la Nación considere prioritarias, y que podrían ser:

- nanobiomedicina,

- energías,

- agricultura, bosques, selvas,

- agua,

- nanomateriales derivados de los minerales extraídos de nuestras minas. 